\title{
Exercise Training Increased S-Klotho Expression on Endothelial Dysfunction: Review
}

\author{
Moran Saghiv ${ }^{1}$ and TJ Exford ${ }^{2 *}$ \\ ${ }^{1}$ Department of Kinesiology, North Carolina Agricultural and Technical State University, USA \\ ${ }^{2}$ Department of Kinesiology, North Carolina Agricultural and Technical State University, USA
}

*Corresponding author: TJ Exford, Department of Kinesiology, North Carolina Agricultural and Technical State University, USA.

Received Date: July 15, 2020

Published Date: August 05, 2020

\begin{abstract}
Endothelial dysfunction is characterized by reduced vascular nitric oxide levels, that causes some irregularities in blood artery function. These functional irregularities contribute to atherosclerosis, which promote vasoconstriction of the small arteries, a major factor in hypertension. Secreted klotho (s-klotho) regulates nitric oxide production in the endothelium. It has been suggested that s-Klotho affect nitric oxide thus benefiting endothelial function. S-klotho levels are reflective of aerobic fitness level, s-Klotho is significantly higher in trained individuals compared to untrained. This suggests aerobic training may be an appropriate model for examining the role of physical activity on s-Klotho expression. There is an association with endothelial fusnction improvement with increased s-klotho levels following aerobic exercise training. It is believed that this review will inspire more deliberation on the relationship between aerobic exercise training and circulating s-Klotho, as a possibly effectors on cardiovascular arterial stiffens due to endothelial dysfunction.
\end{abstract}

Keywords: Nitric oxide; Arteriosclerosis; Hypertension; Apoptosis; Oxidative stress; FGF23

\section{Introduction}

Arterial stiffens describes the inflexibility of the arterial wall that occurs as a result of the aging process and arteriosclerosis. Inflammation has a major part in arteriosclerosis progress, so, it is a main donor in large arterial stiffening [1]. Arterial stiffness result in thickening, fibrosis, fragmentation, and loss of elastin fibers and hence, stiffen the arterial wall by causing structural alterations (arteriosclerosis) and artery inflammatory (atheromasias). In brief, atherogenesis results from lipid peroxidation-derived aldehydes oxidized to carboxylic acids. The pro-inflammatory oxidized phospholipids, resulting of the oxidation of low-density lipoprotein and phospholipids including arachidonic acid, formed in the lipoxygenase and myeloperoxidase pathways, these molecules attract and trigger inflammatory cells, such as monocytes, T cells and macrophages. Matrix degradation brings about atherosclerotic as a result of macrophages activation through cytokines, ROS, and proteolytic enzymes. Oxidative stress is also considered to be a key factor in mechanisms of changes in cell function [2]. In addition, indications suggest that the aging process is in oxidative stress condition [3]. When oxygen is present in large amounts in the cell, reactive oxygen species (ROS) production is increased therefore, the cell needs to eliminate those molecules. ROS molecules have an important part in vascular disease and are known to be a key donor to cardiovascular dysfunction. ROS is produced in large amounts by the enzyme nicotinamide adenine dinucleotide phosphate (NADPH) oxidases [4]. ROS affect other enzymes, such as nitric oxide synthase and endothelial nitric oxide synthase and 
thus, it is a risk factor for atherosclerosis, cardiac hypertrophy, and heart failure. NADPH oxidase activation for the ROS production is facilitated by an extensive number of factors such as hypoxia, cytokines, mechanical forces, and hormones [5]. Cellular ROS are signaling molecules, playing a dominant part as mediators of cellular senescence [6]. Buildup of oxidants might explicate the change of physical work capacity and functions. ROS, including free radicals, is a result of oxygen metabolism and use which, oxidize lipids, DNA, and proteins thus, altering cell's functions [3]. Oxidative stress is the result of endogenous oxygen radicals produced and accumulate in the cells causing damage. Oxidative stress are chemical species containing oxygen, such as peroxides, superoxide, hydroxyl radical and singlet oxygen [7]. Thus, prooxidant stimuli induce apoptotic and nonapoptotic cell death and premature cell senescence in multiple cell types and tissues, which is highly relevant to pathogenesis of atherosclerosis. Previously it has been revealed that cellular senescence has appeared as a significant donor to age-linked disease [8]. Cells certainly produces antioxidants to offset free radicals' destructive effects, but free radicals' production outweighs the number of antioxidants produced. Atherosclerosis reduction and cell protection occur by some antioxidant's such as s-Klotho humoral. In addition, arterial stiffening may predispose the intima to atherosclerosis due to injury sustained from increased pulsatile pressure.

Pressure applied on the blood arteries walls following left ventricle ejection is lowest if the mechanical properties of arteries walls have enough elastance [9]. However, loss of arteries elastance ability, result in arterial stiffness and rise in pulse pressure which in turn increase impedance to left ventricle ejection [10]. The velocity of the pulse wave is related to the stiffness of the arteries. Under these conditions, stroke volume decrease and therefore, only part of cardiac output can be used for oxygen delivery. To compensate, systolic blood pressure and pulse pressure are increased [9]. The vascular endothelium is a monolayer of cells between the vessel lumen and the vascular smooth muscle cells, separates the arterial wall from the blood flow and interacts with circulating blood. The endothelium plays an important role in blood vessel functions by producing various signaling molecules [11]. The endothelium is the internal coating of cells that has several critical functions, it controls the right vessels' radius namely, constriction or dilation, thus changing rapidly blood's amount delivered to the various organs, and blood pressure. The endothelium also protects the tissues from various toxic substances and regulates the a. coagulation which is a mechanism that blood changes from a liquid to a gel, it includes activation, bond, and accumulation of platelets along with deposition and maturation of fibrin, b. controls the fluid, c. regulates electrolytes' levels and, d. exchange of numerous substances between the blood and the tissues and Vic versa, and, regulates inflammation damages in the tissues. Consequently, Endothelium function is critical for the regular function of the arteries, tissues, and organs [12].
Dysregulation of the normal smooth muscle contraction properties response is usually characterized by endothelial dysfunction, following advanced atherosclerosis and increase oxidative stress [13], that induces endothelial dysfunction and atherosclerosis progression by reducing nitric oxide availability [14]. Endothelial dysfunction is mostly triggered by decreased production or action of relaxing mediators. At this point, it is clear that reduction in the levels of nitric oxide cause endothelial dysfunction in blood arterial walls which, leads to vasoconstriction of the artery [15] and thus, increase blood pressure to high values (hypertension). In addition, it also, activates platelets that leads to blood clotting, increases the stimulus of inflammation in artery walls, and increases the penetrability of the arterial walls to destructive lipoproteins, oxidized free radical species and various toxins.

\section{S-Klotho}

Age-related declines and physical inactivity are manifested by a decreased ability for aged skeletal muscle to respond to physiological stimuli such as muscle loading or acute injury. Certainly, older adults often exhibit an age-related reduction in the number and size of muscle fibers known as sarcopenia [16]. Several actions of klotho have been defined to date, Klotho is a putative anti-aging gene, vital cofactor for the linkage of fibroblast growth factor (FGF 23) to its receptor, and thus, acting as a main controller of phosphate balance $[17,18]$. The Klotho proteins and its connected enzyme $\beta$-Klotho apply various influences on the biological regulation of ion transport, energy metabolism, calcium, and phosphate mainly by FGF-23 [19]. In addition, s-Klotho functions as an obligate coreceptor with fibroblast growth factor receptor 1 (FGFR1) for fibroblast growth factor 23 (FGF23), a phosphaturia hormone essential for maintaining mineral homeostasis [20]. Serum FGF23 values are related to atherosclerotic problem, endothelial dysfunction, arterial stiffness, and vascular calcification [21,22]. Circulating s-Klotho is produced in the kidney predominantly expressed in renal distal tubular epithelial cells.

The extracellular domain of Klotho can be cleaved and shed in the circulation as s-Klotho where it may function as a vasculoprotective hormone possibly by enhancing endothelial function $[23,24]$ or direct inhibition of vascular calcification [25]. Klotho appears to apply different functions in distinct cell types in a reliant or, none-reliant means. However, studies on mouse genetic have revealed in vivo functions of Klotho (FGF signaling) [26]. Klotho inhibited the phosphatidylinositol 3-kinase (PI3K)-AKT signaling pathway phosphorylation of fork head box protein 03a by improving its connection to the manganese superoxide dismutase supporter. Klotho increased mitochondrial manganese superoxide dismutase, mRNA, and protein expression. In addition, Klotho reduce tacrolimus-induced oxidative stress and thus, converse mitochondrial dysfunction, consequently, decrease ROS production [27]. Increased cellular levels of ROS result in damage to proteins, 
nucleic acids, lipids, membranes, and organelles, which can lead to activation of cell death processes such as apoptosis. Mice with Klotho-deficient demonstrate an associated with accelerated and enhanced development of vasculopathy [28], and early aging. However, increase Klotho expression increase genetic expression that solve the Klotho-deficient phenotype at baseline [29] and thus, improve mice's standing against oxidant stress [30]. In humans, serum levels of s-Klotho decrease after 40 years of age $[31,32]$. The decrease in blood s-Klotho levels in humans may be observed in sedentary individuals and patients with several aging-related diseases such as cancer, hypertension, and kidney disease [33,34].

\section{Aerobic Exercise}

Physical inactivity decreases maximal oxygen uptake (VO2max) which decrease further with aging [35]. Aging, regardless of lifestyle is impacted by the contribution of genetic factors, which increase the rate of biological processes thereby, severely limiting an older individuals function, life quality, and longevity $[35,36]$. However, endurance exercise training was suggested to reduce rate of decline in work performance typically occurs as an individual age [37]. Apart from genetic endowment, an individual must also interact with environmental factors associated with longevity, by maintaining high level of physical activity [38]. In addition, chronic endurance training attenuates the decline in VO2max associated with age [39]. The basic mechanism by which workout triggers genes (epigenetic) includes a stimulus signal to the DNA, then transcript through messenger RNA, and finally conversion into protein [40].

Previously it has been reported that oxidizing free radical species (ORS) are generated during moderate and high aerobic bouts [41]. Skeletal muscle generates superoxide and nitric oxide during aerobic exercise in intensities above the anaerobic threshold [42]. ROS is essential for skeletal muscle force generation, however, ROS in high values may reduce muscle contraction properties and thus, bring about an early exhaustion [43]. There is plentiful indication that exercise can be operative in averting and suspending the result of age on muscle well-being and effectiveness. Although the association between s-Klotho and aerobic exercise training is not clear, recently, the $\alpha$-Klotho gene is circulating in blood as s-Klotho have been related to the aerobic exercise [44, 45]. Yet, exercise appears to take a major part on the secreted form of the $\alpha$-Klotho gene in humans. In addition, $\alpha$-Klotho gene is associated also with genes: $\beta$-Klotho gene and $\gamma$-Klotho gene, however, the last two mentioned genes do not have any role during exercise [46]. The increase in s-Klotho following aerobic exercise training may be a response to ROS that increase in muscle cells as a result of aerobic training. s-Klotho reduces apoptosis through the nitric oxide production and thus, suppress oxidative stress [47].

\section{Regulations between Exercise, S-Klotho and Endothelial Dysfunction}

Data suggests that endothelium plays a major part in the regulation of arterial stiffness by the action on smooth muscle tone affected by vasoactive intermediaries and, the effect of nitric oxide production on endothelin arterial stiffness [48]. Nitric oxide (nitrogen monoxide) is a soluble gas molecule with the chemical formula for Nitrogen Oxide (NO). It is continually synthesized by the endothelium: Amino acid L-arginine in endothelial cells is the precursor for nitric oxide synthesize by calcium-calmodulindependent enzyme nitric oxide synthase [49]. Nitric oxide acts as an endothelium-derived relaxing factor [50], released from endothelial cells and acts as an inhibitor of ROS production, by decrease in L-arginine endogenous asymmetric dimethyl-Larginine ratio connected with endothelial dysfunction [51]. Nitric oxide stimulates phospholipase A2 and inhibitors of lysolecithin acyltransferase inducing smooth muscle tone relaxation by inhibiting low density lipoprotein oxidation [52]. Klotho has been linked to the prevention of muscle atrophy and cardiovascular disease in aged individuals [28]. Circulating s-Klotho acts as a humoral factor, involved in the endothelium production and regulation of nitric oxide. In turn, nitric oxide protects endothelial penetrability, smooth muscles' contraction by calcium homeostasis and inhibits insulin-like growth factor-1 signaling [52].

Similar anti-aging impacts have also been attributed to aerobic exercise [54,55]. In recent years, there are enough studies regarding the effect of aerobic exercise on blood circulating s-Klotho [31,56]. Reimers et al. [57], demonstrated that the response of s-klotho depends on aerobic fitness level. In addition, levels of s-Klotho were significantly higher in trained individuals compared to untrained once [58], suggesting that long lasting aerobic training may be an appropriate model for mechanistically probing the role of physical activity on s-Klotho expression. The population aged from 0 to 91 years screened previously by ELISA revealed that the level of s-Klotho which is a serum factor related to human declines with aging [59]. Previously in older mice, it has been demonstrated that low blood serum s-Klotho levels are related to reduced skeletal muscle strength and aerobic capacity [60]. On the other hand, trained elderly with aerobic capacity have longer life expectancies [61], and higher serum s-Klotho values compared to inactive elderly [44]. Moderate aerobic training attenuates aging-induced pathological cardiac hypertrophy at least partially by restoring the Klotho levels, attenuating oxidative stress, and reduction in the phosphorylation of ERK1/2, P38 and fibrosis [62]. The relationships between aerobic exercise, s-Klotho and endothelial dysfunction can be in brief described as follow: previously it has been suggested that s-Klotho and long-lasting aerobic exercise training are factors that may promote and upgrade young adults' physical performance capacities [63]. aerobic bout increases s-Klotho levels that in turn, increase FGF23 which promotes NO synthesis bringing about a, reduction in oxidative stress and ROS in skeletal muscle and accordingly, increase mitochondrial vitality [64] and thus, attenuate restore endothelial dysfunction.

\section{Discussion}

So far, the present review, suggests that aerobic exercise increase Klotho gene expression, thus may be a harmless primary 
intervention delay the endothelial dysfunction course and many chronic diseases and cardiovascular-related diseases [65]. In addition, previously it has been shown that moderate aerobic exercise possibly may increase Klotho gene expression in muscle cells and decrease ROD production [44,45,47]. However, others suggested lower aerobic intensities as a way to reduce ROS [66]. Following aerobic exercise training, expression of the membranebound Klotho protein levels was suggestively decreased pointing that membrane-bound Klotho protein compared to the secreted form may be the active one. Recent developments point out the effect of aerobic exercise training program as nitric oxide nonpharmacological means to support the treatment, prevention, and therapy of patients with cardiovascular diseases [67]. The present review suggests that aerobic exercise delays aging process by increase Klotho gene expression, which in turn, reduces ROS damages to the cell. The relationships between the circulating s-Klotho levels a potential anti-aging factor and aerobic exercise are beneficial to physically active aged individuals [68]. In addition, moderate aerobic exercise increase Klotho gene expression in muscle cells and decrease ROD production. Future research should examine the relationship between aerobic exercise training and circulating s-Klotho, on cardiovascular arterial stiffness and endothelial dysfunction.

\section{Acknowledgement}

None.

\section{Conflict of Interest}

The authors attest that we have no affiliations with or involvement in any organization or entity with financial interest (such as honoraria; educational grants; participation in speakers' bureaus; membership, employment, consultancies, stock ownership, or other equity interest; and expert testimony or patentlicensing arrangements), or non-financial interest (such as personal or professional relationships, affiliations, knowledge or beliefs) in the subject matter or materials discussed in this manuscript.

\section{References}

1. Mozos I, Malainer C, Horbańczuk J, Cristina Gug, Dana Stoian, et al. (2017) Inflammatory Markers for Arterial Stiffness in Cardiovascular Diseases. Front Immunol 8: 1058.

2. Finkel T, Holbrook NJ (2000) Oxidants, oxidative stress and the biology of ageing. Nature 408: 239-247.

3. Barouki R (2006) Ageing free radicals and cellular stress. Med Sci (Paris) 22: $266-272$.

4. Frazziano G, Champion HC, Pagano PJ (2012) NADPH oxidase-derived ROS and the regulation of pulmonary vessel tone. Am J Physiol Heart Circ Physiol 302: H2166-H2177.

5. Sahoo S, Meijles ND, Pagano JP (2016) NADPH oxidases: key modulators in aging and age-related cardiovascular diseases? Clin Sci (Lond) 130: 317-335.

6. Colavitti R, Finkel T (2005) Reactive oxygen species as mediators of cellular senescence. IUBMB Life 57: 277-281.

7. Hayyan M, Hashim MA, AlNashef IM (2016) Superoxide Ion: Generation and Chemical Implications. Chem Rev 116: 3029-3085.
8. Childs BG, Durik M, Baker DJ, van Deursen JM (2015) Cellular senescence in aging and age-related disease: from mechanisms to therapy. Nat Med 21: $1424-1435$.

9. Safar ME, Levy BI, Struijker-Boudier H (2003) Current perspectives on arterial stiffness and pulse pressure in hypertension and cardiovascular disease. Circulation 107: 2864-2869.

10. Laurent S, Cockcroft J, Van Bortel L, Pierre Boutouyrie, Cristina Giannattasio, et al. (2006) Expert consensus document on arterial stiffness: methodological issues and clinical applications. Eur Heart J 27: 2588-2605.

11. Rajendran P, Rengarajan T, Thangavel J, Yutaka Nishigaki, Dhanapal Sakthisekaran, et al. (2013) The Vascular Endothelium and Human Diseases. Int J Biol Sci 9: 1057-1069.

12. Godo S, Shimokawa H (2017) Divergent roles of endothelial nitric oxide synthases system in maintaining cardiovascular homeostasis. Free Radic Biol Med 109: 4-10.

13. Wang I, Dong X, Gao J (2018) Targeting Inflammatory Vasculature by Extracellular Vesicles. The AAPS J 20: 37.

14. Modaresi A, Nafar M, Sahraei Z (2015) Oxidative stress in chronic kidney disease. Iran J Kidney Dis 9: 165-179.

15. Vanhoutte PM, Shimokawa H, Feletou M, EHC Tang (2017) Endothelial dysfunction and vascular disease - a 30th anniversary update. Acta Physiol (Oxf) 219: 22-96.

16. Avin KG, Coen PM, Huang W, Donna B Stolz, Gwendolyn A Sowa, et al. (2004) Skeletal muscle as a regulator of the longevity protein, Klotho. Front Physiol 5: 189.

17. Kurosu H, Ogawa Y, Miyoshi M, Yamamoto M, Animesh Nandi, et al. (2006) Regulation of fibroblast growth factor-23 signaling by klotho. J Biol Chem 281: 6120-6123.

18. Urakawa I, Yamazaki Y, Shimada T, Kousuke Iijima, Hisashi Hasegawa, et al. (2006) Klotho converts canonical FGF receptor into a specific receptor for FGF23. Nature 444: 770-774.

19. Long YC, Kharitonenkov A (2011) Hormone-like fibroblast growth factors and metabolic regulation. Biochim Biophys Acta 1812: 791-795.

20. Kuro-o M (2009) Klotho and aging. Biochim Biophys Acta 1790: 10491058.

21. Mirza MA, Hansen T, Johansson L, Håkan Ahlström, Anders Larsson, et al. (2009) Relationship between circulating FGF23 and total body atherosclerosis in the community. Nephrol Dial Transplant 24: 31253131.

22. Mirza MA, Larsson A, Lind L, Tobias E Larsson (2009) Circulating fibroblast growth factor-23 is associated with vascular dysfunction in the community. Atherosclerosis 205: 385-390.

23. van Ark J, Hammes HP, van Dijk MCRF, Chris P H Lexis, Iwan CC van der Horst, et al. (2013) Circulating alpha-klotho levels are not disturbed in patients with type 2 diabetes with and without macrovascular disease in the absence of nephropathy. Cardiovasc Diabetol 12: 116-120

24. Maekawa Y, Ohishi M, Ikushima M, Koichi Yamamoto, Osamu Yasuda et al. (2011) Klotho protein diminishes endothelial apoptosis and senescence via a mitogen-activated kinase pathway. Geriatr Gerontol Int 11: 510-516.

25. Semba RD, Cappola AR, Sun K, Stefania Bandinelli, Mansi Dalal, et al. (2011) Plasma klotho and cardiovascular disease in adults. J Am Geriatr Soc 59: 1596-1601.

26. Shawkat Razzaque M (2012) The role of Klotho in energy metabolism. Nat Rev Endocrinol 8: 579-587.

27. Lim SW, Jin L, Luo K, Jian Jin, Yoo Jin Shin, et al. (2017) Klotho enhances FoxO3-mediated manganese superoxide dismutase expression by negatively regulating PI3K/AKT pathway during tacrolimus-induced oxidative stress. Cell Death Dis 8(8): e2972

28. Kuro-o M, Matsumura Y, Aizawa H, H Kawaguchi, T Suga, et al. (1997) Mutation of the mouse klotho gene leads to a syndrome resembling ageing. Nature 390: 45-51. 
29. Kurosu H, Yamamoto M, Clark JD, Johanne V Pastor, Animesh Nandi, et al. (2005) Suppression of aging in mice by the hormone Klotho. Science 309: 1829-1833.

30. Sugiura H, Yoshida T, Tsuchiya K, Michihiro Mitobe, Sayoko Nishimura, et al. (2005) Klotho reduces apoptosis in experimental ischaemic acute renal failure. Nephrol Dial Transplant 20: 2636-2645.

31. Saghiv SM, Ben Sira D, Goldhammer E, M Sagiv (2017) The effects of aerobic and anaerobic exercises on circulating soluble-Klotho and IGF-I in young and elderly adults and in CAD patients. J Circ Biomark 6: 1849454417733388

32. Matsuda S, Nakagawa Y, Tsuji A, Yasuko Kitagishi, Atsuko Nakanishi, et al. (2018) Implications of PI3K/AKT/PTEN Signaling on Superoxide Dismutases Expression and in the Pathogenesis of Alzheimer's Disease. Diseases 6(2): 28.

33. Wang Y, Sun Z (2009) Current understanding of klotho. Ageing Res Rev 8: 43-51.

34. Wang Y, Sun Z (2014) Antiaging gene Klotho regulates endothelin-1 levels and endothelin receptor subtype B expression in kidneys of spontaneously hypertensive rats. Journal of hypertension 32: 1629 1636

35. Pollock RD, Duggal NA, Lazarus NR, J M Lord, S D R Harridge, et al. (2018) Cardiorespiratory fitness not sedentary time or physical activity is associated with cardiometabolic risk in active older adults. Scand Med Sci Sports 28: 1653-1660

36. Afilalo J, Karunananthan S, Eisenberg MJ, Karen P Alexander, Howard Bergman, et al. (2009) Role of frailty in patients with cardiovascular disease. Am J Cardiol 103: 1616-1621.

37. Nikolaidis PT, Villiger E, Rosemann T, Beat Knechtle (2018) The effect of aging on pacing strategies of cross-country skiers and the role of performance level. Eur Rev Aging Phys Act 15: 4

38. Baldwin KM, Haddad F (2010) Research in the exercise sciences: where we are and where do we go from here. Part II. Exerc Sport Sci Rev 38(2): 42-50.

39. Buford TW, MacNeil RG, Clough LG, Marvin Dirain, Bhanuprasad Sandesara, et al. (2014) Active muscle regeneration following eccentric contraction-induced injury is similar between healthy young and older adults. J Appl Physiol 116: 1481-1490.

40. Puthucheary Z, Skipworth JR, Rawal J, Mike Loosemore, Ken Van Someren, et al. (2011) Genetic influences in sport and physical performance. Sports Med 41: 845-859.

41. McArdle A, Pattwell D, Vasilaki A, RD Griffiths, M J Jackson, et al. (2001) Contractile activity-induced oxidative stress: cellular origin and adaptive responses. Am J Physiol Cell Physiol 280: C621-C627.

42. McArdle A, Pollock N, Staunton CA, Malcolm J Jackson (2018) Aberrant redox signaling and stress response in age-related muscle decline: Role in inter-intra-cellular signaling. Free Radic Biol Med 132: 50-57.

43. Powers KS,Nelson WB, Hudson BM (2011) Exercise-induced oxidative stress in humans: Cause and consequences. Free Radical Biology and Medicine 51: 942-950.

44. Saghiv M, Goldhammer E, Sagiv M (2015) Effects of Aerobic Exercise Training on S-Klotho in Young and Elderly. JJ Physiology 1(1): 001.

45. Amaro-Gahete FJ, de la O A, Jurado-Fasoli L, Andrea Espuch-Oliver, Lidia Robles-Gonzalez, et al. (2018) Exercise training as S-Klotho protein stimulator in sedentary healthy adults: Rationale, design, and methodology. Contemp Clin Trials Commun 11: 10-19.

46. Amaro-Gahete FJ, de la O A, Jurado-Fasoli L, Jonatan R Ruiz, Manuel Castillo, et al. (2018) Role of exercise on S-Klotho protein regulation: a systematic review. Curr Aging Sci 11(2): 100-107.

47. Carracedo J, Buendia P, Merino A, Juan Antonio Madueño, Esther Peralbo, et al. (2012) Klotho modulates the stress response in human senescent endothelial cells. Mech Ageing Dev 133: 647-654.

48. Cannon OR (1998) Role of nitric oxide in cardiovascular disease: focus on the endothelium. Clin Chem 44: 1809-1819.
49. Tousoulis D, Kampoli AM, Tentolouris C, Nikolaos Papageorgiou, Christodoulos Stefanadis (2012) The role of nitric oxide on endothelial function. Curr Vasc Pharmacol 10: 4-18.

50. Palmer RM, Ferrige AG, Moncada S (1987) Nitric oxide release accounts for the biological activity of endothelium-derived relaxing factor. Nature 327: 524-526

51. Engin A (2017) Endothelial Dysfunction in Obesity. Adv Exp Med Biol 960: 345-379.

52. Young SG, Parthasarathy S (1994) Why are low-density lipoproteins atherogenic? West J Med 160: 153-164.

53. Kitagawa M, Sugiyama H, Morinaga $H$, Tatsuyuki Inoue, Keiichi Takiue, et al. (2013) A decreased level of serum soluble Klotho is an independent biomarker associated with arterial stiffness in patients with chronic kidney disease. PLoS One 8(2): e56695

54. Nuria Garatachea, Helios Pareja-Galeano, Fabian Sanchis-Gomar Alejandro Santos-Lozano, Carmen Fiuza-Luces, et al. (2015) Exercise attenuates the major hallmarks of aging. Rejuvenation Res 18(1): 57-89.

55. Amaro-Gahete FJ, de la O A, Jurado-Fasoli L, Jonatan R Ruiz, Manuel J Castillo, et al. (2018) Role of exercise on S-Klotho protein regulation: a systematic review. Curr Aging Sci 11(2): 100-107.

56. Saghiv M, Sherve C, Ben-Sira D (2016) Aerobic training effect on blood S-Klotho levels in coronary artery disease patients. J Clinic Experiment Cardiolog.

57. Reimers CD, Knapp G, Reimers AK (2012) Does physical activity increase life expectancy? A review of the literature. J Aging Res 2012: 243958.

58. Lee EY, Kim SS, Lee JS, In Joo Kim, Sang Heon Song, et al. (2014) Soluble $\alpha$-Klotho as a Novel Biomarker in the Early Stage of Nephropathy in Patients with Type 2 Diabetes. Plos One 9(8): e102984.

59. Crasto CL, Semba RD, Sun K, Anne R Cappola, Stefania Bandinelli, et al (2012) Relationship of low-circulating "anti-aging" klotho hormone with disability in activities of daily living among older community-dwelling adults. Rejuvenation Res 15: 295-301.

60. Avin GK, Dube JJ, Goodpaster HB (2014) Skeletal muscle as a regulator of the longevity protein, Klotho. Front Physiol.

61. Paffenbarger RS Jr, Kampert JB, Lee IM (1997) Physical activity and health of college men: longitudinal observations. Int J Sports Med Suppl: S200-S203.

62. Baghaiee B, Karimi P, Siahkouhian M, Linda S Pescatello (2018) Moderate aerobic exercise training decreases middle-aged induced pathologic cardiac hypertrophy by improving Klotho expression, MAPK signaling pathway, and oxidative stress status in Wistar rats. Iran J Basic Med Sci 21: 911-919.

63. Saghiv M, Ben-Sira D, Sagiv M (2015) Comparison between aerobic and anaerobic training influence on s-klotho blood levels following $60 \mathrm{~min}$ aerobic bout. J J Physiology 1(1): 004

64. Li DJ, Fu H, Zhao T, Min Ni, Fu-Ming Shen (2016) Exercise-stimulated FGF23 promotes exercise performance via controlling the excess reactive oxygen species production and enhancing mitochondrial function in skeletal muscle. Metabolism 65: 747-756.

65. Gibala MJ, Little JP, Macdonald MJ, John A Hawley (2012) Physiological adaptations to low-volume, high-intensity interval training in health and disease. J Physiol 590: 1077-1084.

66. Ji M, Luan J, Hu F, Yirong Zhao, Bosen Lv, et al. (2018) Aerobic exercisestimulated Klotho upregulation extends life span by attenuating the excess production of reactive oxygen species in the brain and kidney. Exp Ther Med 16: 3511-3517.

67. Shirwany AN, Zou NH (2010) Arterial stiffness: a brief review. Acta Pharmacol Sin 31: 1267-1276.

68. Yuechi Xu, Zhongjie Sun (2015) Molecular Basis of Klotho: From Gene to Function in Aging. Endocr Rev 36: 174-193. 